九州大学学術情報リポジトリ

Kyushu University Institutional Repository

\title{
切除不能再発小腸癌患者に対する化学療法における ベバシズマブの上乗せ効果
}

大嶋，琴絵

http://hdl. hand le. net/2324/4474896

出版情報：Kyushu University，2020，博士（医学），論文博士 バージョン：

権利関係: Public access to the fulltext file is restricted for unavoidable reason (3) 
Suggestion of added value by bevacizumab to chemotherapy in patients with unresectable or recurrent small bowel cancer

Kotoe Takayoshi ${ }^{1,2}$, Hitoshi Kusaba ${ }^{1}$, Masato Uenomachi ${ }^{3}$, Kenji Mitsugi ${ }^{3}$, Chinatsu Makiyama $^{4}$, Akitaka Makiyama ${ }^{4}$, Keita Uchino ${ }^{5}$, Tsuyoshi Shirakawa ${ }^{6}$, Yoshihiro Shibata ${ }^{7}$, Yudai Shinohara ${ }^{2}$, Kyoko Inadomi ${ }^{1}$, Kenji Tsuchihashi ${ }^{1}$, Shuji Arita $^{8}$, Hiroshi Ariyama ${ }^{1}$, Taito Esaki $^{2}$, Koichi Akashi ${ }^{1}$, Eishi Baba ${ }^{8}$

1. Department of Medicine and Biosystemic Science, Kyushu University Graduate School of Medical Sciences, Kyushu University, 3-1-1 Maidashi, Higashi-ku, Fukuoka 812-8582, Japan 2. Department of Gastrointestinal and Medical Oncology, National Hospital Organization Kyushu Cancer Center, 3-1-1 Notame, Minami-ku, Fukuoka 811-1395, Japan

3. Department of Oncology, Hamanomachi Hospital, 3-3-1, Nagahama, Chuou-ku, Fukuoka 810-8539, Japan

4. Department of Hematology and Oncology, Japan Community Healthcare Organization Kyushu Hospital, 1-8-1, Kishinoura, Yahatanishi-ku, Kitakyushu 806-8501, Japan

5. Department of Medical Oncology, Clinical Research Institute, National Hospital Organization Kyushu Medical Center, 1-8-1, Jigyouhama, Chuou-ku, Fukuoka 810-0065, Japan 6. Department of Oncology, Miyazaki Prefectural Miyazaki Hospital, 5-30, Kita-takamatsucho, Miyazaki 880-8510, Japan

7. Department of Medical Oncology, Fukuoka Wajiro Hospital, 2-2-75, Wajirooka, Higashi-ku, Fukuoka 811-0213, Japan

8. Department of Comprehensive Clinical Oncology, Faculty of Medical Sciences, Kyushu University, 3-1-1 Maidashi, Higashi-ku, Fukuoka 812-8582, Japan

Corresponding author: Eishi Baba, M.D., Ph.D.

Department of Comprehensive Clinical Oncology, Faculty of Medical Sciences, Kyushu University. 3-1-1 Maidashi, Higashi-ku, Fukuoka 812-8582, Japan

Phone +81-92-642-6921, Fax +81-92-642-6922

Email: e-baba@c-oncology.med.kyushu-u.ac.jp

Keywords: Advanced small bowel adenocarcinoma, chemotherapy, molecular targeting therapy, bevacizumab 


\begin{abstract}
Purpose: Standard therapy for advanced small bowel adenocarcinoma (SBA) has not yet been established. The present study assessed the efficacy and safety of chemotherapy (CT) in association with molecular targeting approaches for SBA.

Methods: The histories of 33 advanced SBA patients from six different institutions in Japan, who received CT from 2008 to 2016, were retrospectively examined for background, clinical course and outcome.

Results: Median patient age was 65 years (range 39-83). Primary tumor was located in the duodenum in 21 patients (67\%), the ampulla of Vater in three patients $(9 \%)$, the jejunum in seven patients $(21 \%)$ and the ileum in one patient (3\%). Histologically, well to moderately- and poorly-differentiated adenocarcinoma were identified in $20(61 \%)$ and nine $(27 \%)$ of patients, respectively. Thirteen patients received a single CT regimen, seven patients received two types of $\mathrm{CT}$ regimen, and 13 patients received three or more $\mathrm{CT}$ regimens. As first-line $\mathrm{CT}$, modified FOLFOX6, capecitabine plus oxaliplatin, and S-1 plus cisplatin were employed in thirteen, one, and four patients, respectively. The response rate (RR) and median progression-free survival (PFS) were 25\% and 6.0 months, respectively. Median overall survival (OS) was 13.0 months. Nine out of the 33 patients received bevacizumab-containing CT and three received cetuximabcontaining CT. Median OS of bevacizumab-containing CT patients was 21.9 months. No unexpected serious adverse events were observed.

Conclusions: The analysis indicates that combination CT for advanced SBA is associated with modest efficacy and safety, and that bevacizumab-containing CT may contribute to favorable outcome in these patients.
\end{abstract}




\section{Introduction}

Malignant neoplasms of the small bowel are rare diseases. They account for less than $3 \%$ of neoplasms of the gastrointestinal tract, and consist of adenocarcinoma, lymphoma, carcinoid and sarcoma [1]. One third of small bowel neoplasms are adenocarcinomas (SBAs), with 56\% of these originating in the duodenum [2]. SBA is relatively difficult to diagnose because of its anatomical location [1], and thus $35-40 \%$ of patients possess distant metastases at initial diagnosis $[3,4]$. Prognosis of SBA is poor, with a 5-year survival rate of $30 \%$ and a median overall survival (OS) of 19 months across all disease stages [5]. Five-year survival of patients with stage IV disease however, is reported to be as low as 3-4\% [5].

While systemic chemotherapy (CT) is generally performed for SBA patients with unresectable or metastatic disease, a standard CT regimen has not yet been established and the CT regimens of advanced colorectal cancer (CRC) and gastric cancer (GC) are thus employed. These regimens, which include 5-fluorouracil, capecitabine, oxaliplatin, cisplatin, and irinotecan, have been based on the results of phase II and retrospective studies, since no randomized, prospective clinical study for advanced SBA has so far been conducted [6-9]. A phase II study examining the combination of capecitabine and oxaliplatin (CAPOX) in 25 advanced SBA patients without prior CT, indicated a response rate (RR) of $52 \%$ and a median OS of 20.4 months [6]. A prospective phase II study involving a modified combination of fluorouracil, oxaliplatin, and leucovorin (mFOLFOX) administered to 33 SBA patients in China, demonstrated a RR of $48.5 \%$ and a median OS of 15.2 months [7]. The combination of fluorouracil, irinotecan, and leucovorin (FOLFIRI) was assessed in a retrospective study of SBA patients resistant to platinum-based CT, and revealed a median OS of 10.5 months, median progression-free survival (PFS) of 3.2 months and a RR of $20 \%$ [8]. Other retrospective studies have also suggested that fluorouracil, platinum and irinotecan may be effective for advanced SBA $[9,10]$.

The clinical benefits of molecularly targeted agents, such as anti-VEGF (vascular endothelial growth factor) antibodies and anti-EGFR (epithelial growth factor receptor) antibodies for advanced CRC, and anti-HER2 (human epidermal growth factor receptor 2) antibody and anti-VEGFR (VEGF receptor) antibody for GC have been demonstrated [11-14]. However, the clinical impacts of a molecularly targeted agent for advanced SBA remain unclear, partly because the molecular characteristics of SBA have not been well described. A previous analysis identified $K R A S$ (v-Ki-ras2 Kirsten rat sarcoma viral oncogene homolog) and $B R A F$ (v-Raf murine sarcoma viral oncogene homolog B) V600E gene mutations in $43 \%$ and 
$2.5 \%$ of SBA tumors, respectively, and HER 2 protein overexpression in $3.2 \%$ of SBA samples [15]. Furthermore, a mismatch repair (MMR) deficiency phenotype was reported in $23 \%$ of SBA patients [15]. These findings suggest that the molecular characteristics of SBA are closer to that of CRC than GC, and that molecularly targeted anti-VEGF and anti-EGFR antibodies might be effective for the treatment of SBA. Administration of the anti-VEGF antibody bevacizumab in SBA patients has been reported in several studies [16,17], however the clinical benefit of adding bevacizumab to $\mathrm{CT}$ has not yet been ascertained. A single retrospective study of four SBA patients has reported that an irinotecan-based CT combined with anti-EGFR antibody (cetuximab) therapy exhibited a favorable efficacy and safety profile [18], but the clinical benefit of this type of approach for SBA remains to be fully evaluated. In the present study, we determined the efficacy and safety of CT in combination with anti-VEGF antibody, for the treatment of unresectable or metastatic SBA in Japan. 


\section{Patients and Methods}

\section{Patients}

A total of 33 patients with advanced SBA who were treated with more than one regimen of systemic CT, were registered between $1^{\text {st }}$ January 2008 and $31^{\text {st }}$ December 2016 in six different institutions of the Kyushu Medical Oncology Group. All patients were 20 years or older, and had histologically proven adenocarcinoma, mucinous adenocarcinoma, signet-ring adenocarcinoma, or adenosquamous carcinoma of the duodenum, jejunum and ileum. Patients with ampullary tumors were also included. Recurrent cases after surgery were required to have a minimum period of 6 months from the last date of administration of adjuvant $\mathrm{CT}$ to the date of confirmation of recurrence. There was no restriction regarding previous therapies for other neoplasms and concurrent active primary cancers. An additional criterion for inclusion was the existence of measurable or evaluable lesions, according to the Response Evaluation Criteria in Solid Tumors guidelines (RECIST ver. 1.1) [19]. The study was approved by the ethics committee of each participating institution, and performed according to the guidelines for biomedical research specified in the Declaration of Helsinki. Because of the retrospective nature of the present study, informed consent was not obtained from each patient.

\section{Clinical variables assessed}

Medical information from each patient was retrospectively examined using electronic records. Items surveyed in this study included age, gender, Eastern Cooperative Oncology Group (ECOG) performance status (PS), primary tumor site (e.g. duodenum, ampullary of Vater, jejunum or ileum), pathohistological diagnosis, KRAS mutation status, EGFR and HER2 tumor expression, metastatic and recurrent sites, and disease status. Data relating to previous treatments, including surgery for curative and non-curative intents, bypass surgery, and adjuvant CT were also assessed. Information gathered for systemic CT included the content of CT, PFS and OS statistics, and the reasons for the termination of initial CT. Therapy-related toxicities were assessed according to the Common Terminology Criteria for Adverse Events (CTC-AE) version 4.0 [20], and toxicities with CTC-AE Grade 3 or worse were surveyed.

\section{Statistical analysis}

PFS was defined as the period from the initiation of therapy to the day of tumor progression determined by each institution, or death from any cause. OS was defined as the period from initiation of CT to the day of death from any cause. The Kaplan-Meier method was used to plot 
PFS and OS, with the log-rank test used to assess differences in survival. Correlations between OS and clinical characteristics were analyzed by Spearman's rank correlation analysis and stepwise multivariate regression analysis. The following factors were examined in univariate analysis of OS: age, gender, ECOG PS, tumor location, histological diagnosis, KRAS status, disease status, metastatic sites, prior primary tumor resection, serum CEA concentration, serum CA19-9 concentration, and concurrent cancer. Multivariate analysis included factors potentially predictive for the risk of death in univariate analysis. The Mann-Whitney $U$ test was used to analyze the difference in the characteristics of the patients who were treated with bevacizumabcontaining $\mathrm{CT}$ and those who were treated without bevacizumab. All analyses were two-tailed, with $\mathrm{p}<0.05$ considered significant. All statistical procedures were performed using SPSS Statistics software version 21 (IBM Japan, Tokyo, Japan). 


\section{Results}

\section{Patient background}

Thirty-three advanced SBA patients who received CT were assessed. Their median age was 65 years (range, 39-83 years), and the population included 24 men $(73 \%)$ and nine women $(27 \%)($ Table 1). ECOG PS was recorded as ' 0 ' in eleven patients $(33 \%)$, ' 1 ' in sixteen patients $(49 \%)$ and ' 2 ' in six patients (18\%). Tumors were located in the duodenum in 21 patients (67\%), the ampulla of Vater in three patients (9\%), the jejunum in seven patients $(21 \%)$, and the ileum in one patient (3\%). Thirty-two patients $(97 \%)$ were diagnosed with adenocarcinoma, and one patient (3\%) with adenosquamous carcinoma. Twenty-two patients $(67 \%)$ had incurable disease at the initial diagnosis of SBA, and 11 patients (33\%) had postoperative recurrence. Among the 23 patients who had prior surgery, 11 received curative surgery and 13 received palliative surgery including bypass surgery. Six patients were treated with surgery followed by adjuvant $\mathrm{CT}$ with either modified FOLFOX6 (mFOLFOX6; fluorouracil, leucovorin, and oxaliplatin) (three patients), CAPOX (one patient), UFT (tegafur-uracil) plus leucovorin (two patients), or S-1 (one patient). Metastasized organs included the liver (12 patients, 36\%), the lung (three patients, 9\%), and the peritoneum (17 patients, 52\%). KRAS exon 2 mutation status was examined in 18 patients, and was found to be mutated in ten of these cases (56\%). Eight patients were examined for EGFR-expression, with seven of these being EGFR-positive and one patient being EGFR-negative. Five patients were examined for HER2-expression, all of which were HER2-negative. Serum concentrations of CEA (carcinoembryonic antigen) were equal to or less than $5 \mathrm{ng} / \mathrm{mL}$ in 24 patients (73\%), and greater than $5 \mathrm{ng} / \mathrm{mL}$ in eight patients (24\%); those of CA19-9 (carbohydrate antigen 19-9) were equal to or less than $37 \mathrm{U} / \mathrm{mL}$ in 19 patients (59\%), and greater than $37 \mathrm{U} / \mathrm{mL}$ in 13 patients (39\%). Six patients (18\%) harbored concurrent cancers.

\section{Efficacies of first-line chemotherapies}

First-line CT regimens for the 33 SBA patients were as follows; mFOLFOX6 in thirteen patients (39\%), mFOLFOX6 plus bevacizumab in one patient (3\%), CAPOX in one patient (3\%), and CAPOX plus bevacizumab in three patients (9\%); FOLFIRI, irinotecan plus cetuximab, and sLV5FU2 (fluorouracil and leucovorin), were each administered to a single patient; S-1 plus cisplatin was administered to four patients (12\%), and S-1 alone to a further four patients (12\%); gemcitabine plus cisplatin was administered to two patients $(6 \%)$, gemcitabine plus S-1 to one patient (3\%), and gemcitabine alone to one patient (3\%). In the 
total cohort, $\mathrm{CT}$ was reported as having been terminated due to progressive disease (PD) in 28 patients (85\%), decreased PS in one patient (3\%), and adverse events in one patient (3\%). In one patient CT was terminated for curative surgery, and in another patient, for curative radiotherapy. In one individual the reason for termination of chemotherapy was unknown.

In total, 20 patients with a measurable lesion were evaluable for treatment efficacy. Five patients (25\%) achieved partial response (PR), seven (35\%) showed stable disease (SD), and eight (40\%) showed progressive disease (PD). The objective RR was $25 \%$, and the disease control rate (complete response $(\mathrm{CR})+\mathrm{PR}+\mathrm{SD}$ ) was $60 \%$. Median PFS of the total patients was 6.0 months (Figure 1A), and median OS was 13.0 months (Figure 1B).

In the univariate analysis of clinical characteristics affecting OS, histological diagnosis $(\mathrm{p}=0.020), K R A S$ gene mutation status $(\mathrm{p}=0.047)$ and prior primary tumor resection $(\mathrm{p}=0.008)$ were found to be associated with improved OS (Table 2). Multivariate analysis was then performed for these three factors, with prior primary tumor resection $(\beta=0.581, p=0.012)$ again being significantly associated with improved OS (Table 3).

\section{Therapy and survival of patients treated with molecular targeted therapy}

Eleven out of the 33 SBA patients (33\%) received the molecularly targeted agents bevacizumab (nine patients; 27\%) or cetuximab (three patients; 9\%). The nine bevacizumab patients were treated with this agent in the first, second and third treatment lines in combination with mFOLFOX6, CAPOX or FOLFIRI (Table 4). Three patients achieved PR following bevacizumab-containing $\mathrm{CT}$, one patient demonstrated $\mathrm{SD}$, and one patient demonstrated non$\mathrm{CR} /$ non-PD. Median OS of the nine patients who had received bevacizumab in any of the treatment lines (the bevacizumab-treated group) was 21.9 months, while median OS of the 24 patients treated without bevacizumab (the bevacizumab-untreated group) was 11.4 months $(\mathrm{p}=0.179)$ (Figure 1C). The clinical characteristics of patients who received bevacizumabcontaining CT and those who received CT without bevacizumab were compared (Table 5). Histological diagnosis was significantly different between the groups $(\mathrm{p}=0.041)$, but no other significant differences were found between the groups. Grade 3 or 4 adverse events associated with bevacizumab were observed only in a single patient, reported as a grade 3 rectal fistula during treatment with CAPOX plus bevacizumab.

Three patients with $K R A S$ wild-type tumors received cetuximab in the first and the third treatment line. Two patients were treated with cetuximab plus irinotecan, and one patient 
was treated with cetuximab alone. Two patients achieved SD in response to cetuximabcontaining CT.

\section{Treatment after first-line CT}

Twenty patients received second-line or additional CT regimens. The second-line CT regimens were as follows: FOLFIRI in five patients, FOLFIRI plus bevacizumab in three patients, irinotecan plus S-1 in two patients, irinotecan alone in two patients, and paclitaxel in four patients; mFOLFOX6, SOX (S-1 and oxaliplatin) plus bevacizumab, S-1 alone, and gemcitabine plus cisplatin, were each administered to a single patient. The median PFS of second-line CT in these patients was 2.5 months. CT treatment beyond third-line was administered to a total thirteen patients. 


\section{Discussion}

A standard CT approach based on large-scale randomized clinical studies has not yet been established for advanced SBA. Recent prospective phase II studies have shown modest efficacy of first line CAPOX [6], mFOLFOX [7], and CAPOX plus bevacizumab [16] for advanced SBA, with median OS of 15-20 months. A retrospective review of CT efficacy in 93 cases of advanced SBA demonstrated a median PFS of 6.6 months and a median OS of 15.1 months [10]. Although the survival benefits for SBA were slightly poorer than those seen in metastatic CRC [21], combinations of oxaliplatin and fluoropyrimidines have been utilized for advanced SBA based on these findings. It is relevant to note that in the study employing CAPOX [6], 12 of the 30 patients (i.e. $40 \%$ ) harbored ampullary adenocarcinoma (AAC) and their RR appeared lower than the rate for SBA (33\% versus 61\%) [6]. In the present study, in which only $9 \%$ of the patients had AAC, modest survival benefits were shown, with a median OS of 13.0 months and a median PFS of 6.0 months. The lower proportion of AAC patients in our study might be one of the reasons for more favorable survival data than that seen in the previous phase II study.

Aparicio et al. previously reported that SBA stages I-II, WHO (World Health Organization) PS 0-1, and a MMR-deficiency phenotype, were correlated with longer OS for all patients, and that PS 0-1 and KRAS mutation predicted a longer OS for stage IV patients [15]. Other studies have shown that older age, higher tumor stage, poor tumor differentiation, positive resection margins, lymphovascular invasion, lymph node invasion and a low number of recovery lymph nodes, are correlated with poor prognosis [22-24]. Among SBAs, duodenal tumors have been reported to have a worse prognosis than jejunal or ileal tumors [3,5]. In our study, $64 \%$ of patients had duodenal tumors, $30 \%$ had non-KRAS mutated tumors, and $18 \%$ had tumors with PS 2, suggesting a relatively unfavorable patient background.

While $K R A S$ mutation is identified in around $40 \%$ of CRC patients, it is associated with only $3-10 \%$ of patients with GC [25-27]. On the other hand, tumor overexpression of HER 2 protein is found in around $15 \%$ of GC patients, but only 2-3\% of CRC patients. Although SBA is generally thought to be a heterogeneous cancer, the high incidence of $K R A S$ mutation and rarity of HER2-overexpression suggests that SBA may possess a similar genetic background to that of CRC [15]. In the present study, none of the patients that were examined for HER2 expression showed overexpression of the protein, and $56 \%$ of the patients that were assessed for $K R A S$ status demonstrated mutation, suggesting that most patients exhibited this previously-reported CRC-like genetic background [15]. 
SBA often arises in the genetic context of Lynch syndrome [28,29]. A previous study reported that 14 out of 61 SBA patients were identified with MMR-deficiency, and of these, nine were diagnosed with Lynch syndrome [15]. Six out of 33 patients (18\%) in our study harbored concurrent cancers, including gastric, colorectal, bladder, gall bladder, ovary, endometrial, and ureteral cancers, and liposarcoma, which (with the exception of liposarcoma) are often observed in patients with Lynch syndrome. One out of these six patients fulfilled the Amsterdam II criteria for the diagnosis of Lynch syndrome [30], while the other five patients possibly have Lynch syndrome with simultaneous occurrence of rare SBA. Since no information regarding MMR genetic alterations or MMR instability in these patients was available, the exact genetic background of these cases cannot be determined. The median OS of the six patients was 10.8 months, which is better than the overall median survival in this study. While SBA with MMR deficiency has been reported to have favorable prognosis [15], concurrent cancer could influence unfavorably on the treatment results for SBA.

Advanced SBA has often been treated with a combination of platinum plus fluorouracil or irinotecan, CT regimens that are employed for both CRC and GC. However, molecularly targeted agents have also been used in the treatment of these diseases. Trastuzumab for HER2-overexpressed GC, and ramucirumab for the second line CT of GC are utilized, but efficacy of bevacizumab in GC has not been proven [31]. The anti-EGFR antibodies, cetuximab and panitumumab, are known to be beneficial for KRAS wild-type CRC patients, but not for GC patients. Bevacizumab and ramucirumab are often employed for CRC. Finally, the efficacy of anti-HER2 therapy for HER2-overexpressing CRC has been reported in a phase II clinical trial [32]. However, the effectiveness of these targeted agents in SBA has not been sufficiently evaluated.

Gulhati et al. reported a phase II study evaluating the benefit of adding bevacizumab to CAPOX in patients with SBA and AAC. The median PFS and OS were 8.7 months and 12.9 months, respectively. They compared these results with their previous data of 25 patients in a phase II study, but were unable to determine significant benefits on RR and PFS because of the retrospective nature of the study and the small size of the patient cohort. Finally, the authors concluded that CAPOX with bevacizumab was an active and well-tolerated regimen for patients with SBA and AAC [16].

On the other hand, Aydin et al. reported that the combination of bevacizumab with mFOLFOX6 or FOLFIRI, produced no significant difference in PFS and OS compared with CT alone, in patients with SBA. The median PFS was 7.7 and 9.6 months in the CT alone and the 
bevacizumab-containing CT groups, respectively, and the median OS was 14.8 and 18.5 months, respectively [17]. In contrast, several case reports have shown efficacy of bevacizumab-containing CT for SBA [33,34]. Bevacizumab inhibits tumor angiogenesis by specifically binding to VEGF-A. In a study examining VEGF-A expression in 54 SBA patients, $96 \%$ of samples were found to be positive [35], suggesting the potential utility of bevacizumab in the treatment of SBA. However, to date, the efficacy of bevacizumab in SBA remains to be proven.

Adverse events, including bleeding and gastrointestinal tract perforation, have been shown for bevacizumab-containing CT in various cancers. In one study, $0.9 \%$ of CRC patients treated with bevacizumab showed gastrointestinal tract perforation, from which the mortality rate was $21.7 \%$ [36]. Known risk factors for perforation include age $\leq 65$ years, no primary tumor resection, and a history of preoperative radiotherapy [37]. Bowel obstruction has also been suggested as a bevacizumab-induced severe adverse event in the patients with ovarian cancer [38]. Since common symptoms of SBA include bowel obstruction and bleeding, bevacizumab treatment may increase the risk of appearance of these symptoms.

In this study, we examined the response of nine SBA patients who had CT in combination with bevacizumab, and observed a more favorable OS than patients treated without bevacizumab. Due to limited patient numbers and the retrospective nature of this study, consideration needs to be given to the possibility of bias in regards to patients with a relatively better condition being preferentially treated with bevacizumab. We thus compared the patient groups treated with or without bevacizumab, thereby only histological diagnosis was significantly different between the groups in their clinical characteristics. More patients with poorly differentiated adenocarcinoma were included in the bevacizumab-untreated group than in the bevacizumab-treated group (33\% vs. $11 \%$ ). We found that surgical resection of the primary tumor was performed more often in the bevacizumab-treated group than in the bevacizumabuntreated group (56\% vs. $42 \%$ ), and that the numbers of patients with ECOG PS 2, and a tumor location in the ampulla of Vater, were less in the bevacizumab-treated group than in the bevacizumab-untreated group, factors that might reflect on prognosis. Seven patients received bevacizumab in second or third line therapies, with PR and SD each observed in one patient, an observation that suggests there may be a modest efficacy of bevacizumab-containing $\mathrm{CT}$ in the second or third line treatment of SBA.

Multivariate analysis demonstrated that prior surgical resection of the primary tumor was a favorable factor of better survival in this study. Bevacizumab-containing CT was 
administered more often to patients with prior surgical resection of the primary tumor, than those with non-primary resection. Despite this possible bias, the observation suggests that CT in combination with bevacizumab might be beneficial for metastatic SBA. Cetuximab was administered to three $K R A S$ wild-type SBA patients in our study, with subsequent SD demonstrated in two of these cases, but because of the small patient population, the survival benefit of this anti-EGFR antibody could not be formally determined. An ongoing phase II clinical study assessing the safety and_efficacy of CAPOX plus the anti-EGFR antibody panitumumab, for KRAS wild-type SBA and AAC (NCT01202409) will help to determine the clinical benefit of this type of therapeutic approach.

In conclusion, the findings from this retrospective study indicate a potential survival benefit of platinum plus fluoropyrimidine combination therapy for advanced SBA. Moreover, bevacizumab-containing CT was found to be beneficial for these patients. Even though SBA is a rare cancer, continued investigation of the efficacy of molecularly targeted drugs for this disease is warranted.

\section{Acknowledgements}

The authors thank the patients and their families for participating in this study, and the medical staff for their respective contributions to the treatment of patients.

\section{Compliance with ethical standards}

\section{Conflict of interests}

Baba E has received research grants from Chugai and Merck Serono. Akashi K has received research grants from Chugai, Yakult and Merck Serono and an honorarium from Chugai. Esaki $\mathrm{T}$ has received a research grant from Merck Serono and honoraria from Chugai and Merck Serono.

\section{Ethical standards}

The study was performed in accordance with the ethical standards laid down in the 1964 Declaration of Helsinki and its later amendments. The study was approved by the ethics committee of each participating institution. For this type of study formal consent is not required. 


\section{References}

1. Radhav K, Overman MJ (2013) Small bowel adenocarcinoma-existing evidence and evolving paradigm Nat Rev Clin Oncol 10:534-544.

2. Bilimoria KY, Bentrem DJ, Wayne JD, Ko CY, Bennett CL, Talamonti MS (2009) Small bowel cancer in the United States: changes in epidemiology, treatment, and survival over the last 20 years. Ann Surg .249:63-71. doi: 10.1097/SLA.0b013e31818e4641

3. Dabaja BS, Suki D, Pro B, Bonnen M, Ajani J (2004) Adenocarcinoma of the small bowel: presentation, prognostic factors, and outcome of 217 patients. Cancer 101:518-26

4. Talamonti MS, Goetz LH, Rao S, Joehl RJ (2002) Primary cancers of the small bowel: analysis of prognostic factors and results of surgical management. Arch Surg 137:564-570

5, Howe JR, Karnell LH, Menck HR, Scott-Conner C (1999) The American College of Surgeons Commission on Cancer and the American Cancer Society. Adenocarcinoma of the small bowel: review of the National Cancer Data Base, 1985-1995. Caner 86:2693-2706 6. Overman MJ, Varadhachary GR, Kopetz S, Adinin R, Lin E, Morris JS, Eng C, Abbruzzese JL, Wolff RA (2009) Phase II study of capecitabine and oxaliplatin for advanced adenocarcinoma of the small bowel and ampulla of Vater. J Clin Oncol 27:2598-2603. doi: 10.1200/JCO.2008.19.7145

7. Xiang XJ, Liu YW, Zhang L, Qiu F, Yu F, Zhan ZY, Feng M, Yan J, Zhao JG, Xiong JP (2012) A phase II study of modified FOLFOX as first-line chemotherapy in advanced small bowel adenocarcinoma. Anticancer Drugs 23:561-566. doi: 10.1097/CAD.0b013e328350dd0d 8. Zaanan A, Gauthier M, Malka D, Locher C, Gornet JM, Thirot-Bidault A, Tougeron D, Taïeb J, Bonnetain F, Aparicio T (2011) Second-line chemotherapy with fluorouracil, leucovorin, and irinotecan (FOLFIRI regimen) in patients with advanced small bowel adenocarcinoma after failure of first-line platinum-based chemotherapy: a multicentre AGEO study. Cancer 117:14221428. doi: 10.1002/cner.25614

9. Tsushima T, Taguri M, Honma Y et al (2012) Multicenter retrospective study of 132 patients with unresectable small bowel adenocarcinoma treated with chemotherapy. Oncologist 17:11631170

10. Zaanan A, Costes L, Gauthier M et al (2010) Chemotherapy of advanced small-bowel adenocarcinoma: a multicenter AGEO study. Ann Oncol 21:1786-1793. doi: 10.1093/annonc/mdq038 11. Douillard JY, Siena S, Cassidy J et al (2010) Randomized, phase III trial of panitumumab with infusional fluorouracil, leucovorin, and oxaliplatin (FOLFOX4) versus FOLFOX4 alone as 
first-line treatment in patients with previously untreated metastatic colorectal cancer: the PRIME study. J Clin Oncol 28:4697-4705. doi: 10.1200/JCO.2009.27.4860

12. Saltz LB, Clarke S, Díaz-Rubio E et al (2008) Bevacizumab in combination with oxaliplatin-based chemotherapy as first-line therapy in metastatic colorectal cancer: a randomized phase III study. J Clin Oncol 26:2013-2019. doi: 10.1200/JCO.2007.14.9930. 13. Bang YJ, Van Cutsem E, Feyereislova A et al (2010) ToGA Trial Investigators. Trastuzumab in combination with chemotherapy versus chemotherapy alone for treatment of HER2-positive advanced gastric or gastro-oesophageal junction cancer (ToGA): a phase 3, open-label, randomized controlled trial. Lancet 376:687-697. doi: 10.1016/S01406736(10)61121-X

14. Wilke H, Muro K, Van Cutsem E et al (2014) RAINBOW Study Group. Ramucirumab plus paclitaxel versus placebo plus paclitaxel in patients with previously treated advanced gastric or gastro-oesophageal junction adenocarcinoma (RAINBOW): a double-blind, randomised phase 3 trial. Lancet Oncol 15:1224-1235. doi: 10.1016/S1470-2045(14)70420-6

15. Aparicio T, Svrcek M, Zaanan A et al (2013) Small bowel adenocarcinoma phenotyping, a clinicobiological prognostic study. Br J Cancer 109:3057-3061. doi: 10.1038/bjc.2013.677. 16. Gulhati P, Raghav K, Shroff RT et al (2017) Bevacizumab combined with capecitabine and oxaliplatin in patients with advanced adenocarcinoma of the small bowel or ampulla of vater: A single-center, open-label, phase 2 study. Cancer 123:1011-7

17. Aydin D, Sendur MA, Kefeli U et al (2016) Evaluation of Bevacizumab in Advanced Small Bowel Adenocarcinoma. Clin Corolectal Cancer doi: 10.1016/j.clcc.2016.04.013.

18. Santini D, Fratto ME, Spoto C, Russo A, Galluzzo S, Zoccoli A, Vincenzi B, Tonini G (2010) Cetuximab in small bowel adenocarcinoma: a new friend? Br J Cancer 103:1305. doi: 10.1038/sj.bjc. 6605898

19. Eisenhauer EA, Therasse P, Bogaerts J et al (2009) New response evaluation criteria in solid tumours: revised RECIST guideline (version 1.1). Eur J Cancer 45:228-247. doi: 10.1016/j.ejca.2008.10.026.

20. National Cancer Institute: Common Terminology Criteria for Adverse Events (CTCAE), version 4.0. http://evs.nci.nih.gov/ftp1/CTCAE/CTCAE_4.03_2010-06-

14_QuickReference_5x7.pdf. Accessed February 10, 2017.

21. Cassidy J, Clarke S, Díaz-Rubio E et al (2008) Randomized phase III study of capecitabine plus oxaliplatin compared with fluorouracil/folinic acid plus oxaliplatin as first-line therapy for metastatic colorectal cancer. J Clin Oncol 26:2006-12. 
22. Halfdanarson TR, McWilliams RR, Donohue JH, Quevedo JF (2010) A single-institution experience with 491 cases of small bowel adenocarcinoma. Am J Surg 199:797-803. doi: 10.1016/j.amjsurg.2009.05.037

23. Nicholl MB, Ahuja V, Conway WC, Vu VD, Sim MS, Singh G (2010) Small bowel adenocarcinoma: understaged and undertreated? Ann Surg Oncol 17:2728-2732. doi: $10.1245 / \mathrm{s} 10434-010-1109-\mathrm{x}$

24. Overman MJ, Hu CY, Wolff RA, Chang GJ (2010) Prognostic value of lymph node evaluation in small bowel adenocarcinoma: analysis of the surveillance, epidemiology, and end results database. Cancer 116:5374-5382. doi: 10.1002/cncr.25324

25. Kim IJ, Park JH, Kang HC, Shin Y, Park HW, Park HR, Ku JL, Lim SB, Park JG (2003) Mutational analysis of BRAF and K-ras in gastric cancers: absence of BRAF mutations in gastric cancers. Hum Genet 114:118-120

26. Moehler M, Mueller A, Trarbach T, Lordick F, Seufferlein T, Kubicka S, Geissler M, Schwarz S, Galle PR (2011) Cetuximab with irinotecan folinic acid and 5-fluorouracil as firstline treatment in advanced gastroesophageal cancer: a prospective multi-center biomarkeroriented phase II study. Ann Oncol 22:1358-1366. doi: 10.1093/annonc/mdq591 27. Lordick F, Luber B, Lorenzen S et al (2010) Cetuximab plus oxaliplatin/leucovorin/5fluorouracil in first-line metastatic gastric cancer: a phase II study of the Arbeitsgemeinschaft Internistische Onkologie (AIO) Br J Cancer 102:500-505. doi: 10.1038/sj.bjc.6605521 28. Koornstra JJ, Kleibeuker JH and Vasen HF (2008) Small-bowel cancer in Lynch syndrome: Is it time for surveillance? Lancet Oncol 9:901-905. doi: 10.1016/S1470-2045(08)70232-8 29. Watson P, Vasen HF, Mecklin JP, Bernstein I, Aarnio M, Järvinen HJ, Myrhøj T, Sunde L, Wijnen JT, Lynch HT (2008) The risk of extra-colonic, extra-endometrial cancer in the Lynch syndrome. Int J Cancer 123:444-449. doi: 10.1002/ijc.23508

30. Vasen HF, Watson P, Mecklin JP, Lynch HT (1999) New clinical criteria for hereditary nonpolyposis colorectal cancer (HNPCC, Lynch syndrome) proposed by the International Collaborative Group on HNPCC. Gastroenterology 116:1453-1456

31. Ohtsu A, Shah MA, Van Cutsem E et al (2011) Bevacizumab in combination with chemotherapy as first-line therapy in advanced gastric cancer: a randomized, double-blind, placebo-controlled phase III study. J Clin Oncol 29:3968-3976. doi: 10.1200/JCO.2011.36.2236 32. Sartore-Bianchi A, Trusolino L, Martino C et al. (2016) Dual-targeted therapy with trastuzumab and lapatinib in treatment-refractory, KRAS codon 12/13 wild-type, HER2positive metastatic colorectal cancer (HERACLES): a proof-of-concept, multicentre, open-label, phase 2 trial. Lancet Oncol 17:738-746. doi: 10.1016/S1470-2045(16)00150-9 
33. Nagaraj G, Zarbalian Y, Flora K, Tan BR Jr (2014) Complete response and prolonged disease-free survival in a patient with recurrent duodenal adenocarcinoma treated with bevacizumab plus FOLFOX6. J Gastrointest Oncol 5:E1-6. doi: 10.3978/j.issn.20786891.2013 .038

34. Okubo K, Yoshioka S, Asukai K et al (2010) A case report of primary adenocarcinoma of small intestine. Gan To Kagaku Ryoho 37:2792-2794

35. Overman MJ, Pozadzides J, Kopetz S, Wen S, Abbruzzese JL, Wolff RA, Wang H (2010) Immunophenotype and molecular characterisation of adenocarcinoma of the small intestine. $\mathrm{Br}$ J Cancer 102:144-150. doi: 10.1038/sj.bjc.6605449

36. Hapani S, Chu D, Wu S (2009) Risk of gastrointestinal perforation in patients with cancer treated with bevacizumab: a meta-analysis. Lancet Oncol 10:559-568. doi: 10.1016/S14702045(09)70112-3

37. Kozloff M, Yood MU, Berlin J, Flynn PJ, Kabbinavar FF, Purdie DM, Ashby MA, Dong W, Sugrue MM, Grothey A (2009) Investigators of the BRiTE study. Clinical outcomes associated with bevacizumab-containing treatment of metastatic colorectal cancer: the BRiTE observational cohort study. Oncologist 14:862-870. doi: 10.1634/theoncologist.2009-0071 38. Garcia AA, Hirte H, Fleming G et al (2008) Phase II clinical trial of bevacizumab and lowdose metronomic oral cyclophosphamide in recurrent ovarian cancer: a trial of the California, Chicago, and Princess Margaret Hospital phase II consortia. J Clin Oncol 26:76-82. doi: 10.1200/JCO.2007.12.1939 
Table 1. Patient characteristics

$\begin{array}{lll}\text { Characteristics } & \text { No. of patients } & \%\end{array}$

(Total $\mathrm{n}=33$ )

Age, years
Median (Range)
$65(39-83)$

Gender

$\begin{array}{lll}\text { Male } & 24 & 73\end{array}$

$\begin{array}{lll}\text { Female } & 9 & 27\end{array}$

ECOG performance status

$\begin{array}{lll}0 & 11 & 33 \\ 1 & 16 & 49 \\ 2 & 6 & 18\end{array}$

Tumor location

$\begin{array}{llc}\text { Duodenum } & 21 & 67 \\ \text { Ampulla of Vater } & 3 & 9 \\ \text { Jejunum } & 7 & 21 \\ \text { Ileum } & 1 & 3 \\ \text { Small bowel NOS } & 1 & 3\end{array}$

Histological diagnosis

$\begin{array}{lll}\text { Adenocarcinoma } & 32 & 97\end{array}$

$\begin{array}{lll}\text { Well to moderately differentiated } & 20 & 61\end{array}$

$\begin{array}{lll}\text { Poorly differentiated } & 9 & 27\end{array}$

Not specified $\quad 3 \quad 9$

Adenosquamous carcinoma $\quad 1 \quad 3$

$K R A S$ gene status

$\begin{array}{lll}\text { Wild-type } & 8 & 24\end{array}$

$\begin{array}{lll}\text { Mutant type } & 10 & 30\end{array}$

$\begin{array}{lll}\mathrm{N} / \mathrm{E} & 15 & 46\end{array}$

EGFR expression status

$\begin{array}{lll}\text { Positive } & 7 & 21 \\ \text { Negative } & 1 & 3 \\ \text { N/E } & 25 & 76\end{array}$

HER2 status

Positive 00

$\begin{array}{lll}\text { Negative } & 5 & 15\end{array}$

$\begin{array}{lll}\mathrm{N} / \mathrm{E} & 28 & 85\end{array}$

Disease status

$\begin{array}{lll}\text { Unresectable } & 22 & 67\end{array}$

$\begin{array}{lll}\text { Recurrent } & 11 & 33\end{array}$

Metastatic site 


$\begin{array}{lll}\text { Liver } & 12 & 36 \\ \text { Lung } & 3 & 9 \\ \text { Peritoneum } & 17 & 52 \\ \text { Lymph node } & 17 & 52 \\ \text { Bone } & 2 & 6\end{array}$

Prior primary tumor surgery

$\begin{array}{lll}\text { Curative } & 11 & 33 \\ \text { Palliative (bypass) } & 13(9) & 39(27)\end{array}$

Prior adjuvant chemotherapy

$\begin{array}{lll}\text { UFT }+ \text { LV } & 2 & 6 \\ \text { mFOLFOX6 } & 3 & 9 \\ \text { CAPOX } & 1 & 3 \\ \text { S- } 1 & 1 & 3\end{array}$

Serum CEA concentration

$\begin{array}{lll}\leq 5.0 \mathrm{ng} / \mathrm{mL} & 24 & 73 \\ >5.0 \mathrm{ng} / \mathrm{mL} & 8 & 24 \\ \mathrm{~N} / \mathrm{E} & 1 & 3\end{array}$

Serum CA19-9 concentration

$\begin{array}{lll}\leq 37 \mathrm{U} / \mathrm{m} & 19 & 59 \\ >37 \mathrm{U} / \mathrm{mL} & 13 & 39 \\ \mathrm{~N} / \mathrm{E} & 1 & 3\end{array}$

Concurrent cancer

Yes $\quad 6 \quad 18$

No $\quad 27 \quad 82$

ECOG, Eastern cooperative oncology group; KRAS, v-Ki-ras2 Kirsten rat sarcoma viral oncogene homolog; EGFR, Epidermal growth factor receptor; HER2, human epidermal growth factor receptor 2; N/E, not examined; UFT, tegafur uracil; LV, leucovorin; mFOLFOX6, modified fluorouracil, leucovorin, and oxaliplatin; CAPOX, capecitabine and oxaliplatin; CEA, carcinoembryonic antigen; CA19-9, carbohydrate antigen 19-9; NOS, not otherwise specified. 
Table 2. Univariate analysis of clinical characteristics and overall survival

\begin{tabular}{lll}
\hline Characteristics & Correlation coefficient & P value \\
\hline Age & 0.017 & 0.926 \\
Gender & 0.171 & 0.340 \\
ECOG performance status & 0.324 & 0.066 \\
Tumor location & 0.117 & 0.516 \\
Histological diagnosis & 0.402 & 0.020 \\
KRAS status (wild-type/mutant) $*$ & 0.474 & 0.047 \\
Disease status & 0.304 & 0.086 \\
Metastatic site & & \\
$\quad$ Liver $\quad$ Peritoneum & 0.198 & 0.268 \\
\multicolumn{1}{c}{ Lymph node } & 0.045 & 0.805 \\
Prior primary tumor resection (yes/no) & 0.204 & 0.255 \\
Serum CEA concentration** & 0.454 & 0.008 \\
Serum CA19-9 concentration** & 0.047 & 0.799 \\
Concurrent cancer (yes/no) & 0.203 & 0.264 \\
\hline
\end{tabular}

${ }^{*} \mathrm{~N}=18 ; * * \mathrm{~N}=32$; Coefficients and p-values derived from Spearman's rank correlation analysis; ECOG, Eastern cooperative oncology group; KRAS, v-Ki-ras2 Kirsten rat sarcoma viral oncogene homolog; CEA, carcinoembryonic antigen $(\leq 5.0 \mathrm{ng} / \mathrm{mL}$ or $>5.0 \mathrm{ng} / \mathrm{mL})$; CA19-9, carbohydrate antigen 19-9 $(\leq 37$ $\mathrm{U} / \mathrm{mL}$ or $>37 \mathrm{U} / \mathrm{mL})$. 
Table3. Multivariate analysis of clinical characteristics and overall survival

\begin{tabular}{lcc}
\hline Items & $\beta$ & P value \\
\hline Histological diagnosis & & 0.253 \\
$K R A S$ status & 0.144 \\
Priory primary tumor resection & 0.581 & 0.012 \\
\hline
\end{tabular}

15 patients were excluded because they had no KRAS status data. Of the remaining patients, 18 were selected for multivariate analysis. The standardized partial regression coefficient $(\beta)$ were calculated by step-wise multivariate regression; KRAS, v-Ki-ras2 Kirsten rat sarcoma viral oncogene homolog. 
Table 4. Therapy and survival of patients treated with bevacizumab-containing therapy

\begin{tabular}{llllll}
\hline Patient & $\begin{array}{l}\text { Treatment } \\
\text { Line }\end{array}$ & $\begin{array}{l}\text { Combination } \\
\text { Chemotherapy }\end{array}$ & Response & $\begin{array}{c}\text { PFS } \\
\text { (months) }\end{array}$ & $\begin{array}{c}\text { OS } \\
\text { (months) }\end{array}$ \\
\hline 1 & 1 st & mFOLFOX6 & PR & 16.9 & 21.8 \\
& 2 nd & FOLFIRI & PD & 2.1 & \\
2 & 1 st & CAPOX & NE & 15.7 & 21.9 \\
& 2nd & SOX & PR & 15.0 & \\
3 & 1 st & CAPOX & PR & 13.4 & 16.7 \\
4 & 1st & CAPOX & Non-CR/Non-PD & - & 36.3 (alive) \\
5 & 2nd & FOLFIRI & SD & 6.2 & 38.0 \\
6 & 2nd & FOLFIRI & PD & 2.5 & 9.2 \\
7 & 3rd & mFOLFOX6 & PD & 1.8 & 13.1 \\
8 & 3rd & FOLFIRI & PD & 5.3 & 29.6 \\
9 & 3rd & FOLFIRI & PD & 1.8 & 14.9 \\
\hline
\end{tabular}

PFS, progression-free survival; OS, overall survival; mFOLFOX6, modified fluorouracil, leucovorin, and oxaliplatin; CAPOX, capecitabine and oxaliplatin; SOX, S-1 and oxaliplatin; FOLFIRI, fluorouracil, leucovorin, and irinotecan; CR, complete response; $\mathrm{PR}$, partial response; $\mathrm{SD}$, stable disease; $\mathrm{PD}$, progressive disease; NE, not examined. 
Table 5. Comparison between patient groups treated with or without bevacizumab

Characteristics

No. of patients

$P$ value

With Bev $(\mathrm{N}=9) \quad$ Without Bev $(\mathrm{N}=24)$

Age, years Median (Range)

$60(48-67)$

$67(39-83)$

0.266

Gender

Male

Female

0.637

ECOG performance status

3

0

1

2

6

18

$4 \quad 7$

$4 \quad 12$

$1 \quad 5$

Tumor location

0.925

Duodenum

Ampulla of Vater

6

15

$0 \quad 3$

Jejunum

25

Ileum

0

1

Small bowel NOS

10

Histological diagnosis

Adenocarcinoma

Well to moderately differentiated

$$
8
$$

12

Poorly differentiated

$$
8
$$

Not specified

1

0

3

Adenosquamous carcinoma

KRAS gene status

$0 \quad 1$

$\begin{array}{lll}\text { Wild-type } & 3 & 5 \\ \text { Mutant type } & 4 & 6\end{array}$

Disease status

Unresectable

Recurrence

5

Metastatic site

Liver
Peritoneum
Lymph node

Prior primary tumor resection

Prior adjuvant chemotherapy

Serum CEA concentration $>5.0 \mathrm{ng} / \mathrm{mL}$

4

17

7

Serum CA19-9 concentration $>37 \mathrm{U} / \mathrm{mL}$

$\begin{array}{lll}4 & 8 & 0.561 \\ 5 & 12 & 0.779 \\ 4 & 13 & 0.624 \\ 5 & 10 & 0.482 \\ 3 & 4 & 0.304 \\ 2 & 6 & 0.870 \\ 4 & 9 & 0.720\end{array}$

The Mann-Whitney U test was used to analyze the difference in the characteristics of the patient groups. Bev, Bevacizumab; ECOG, Eastern cooperative oncology group; KRAS, v-Ki-ras2 Kirsten rat sarcoma viral oncogene homolog; CEA, carcinoembryonic antigen; CA19-9, carbohydrate antigen 19-9; NOS, not otherwise specified. 


\section{Figure.1}
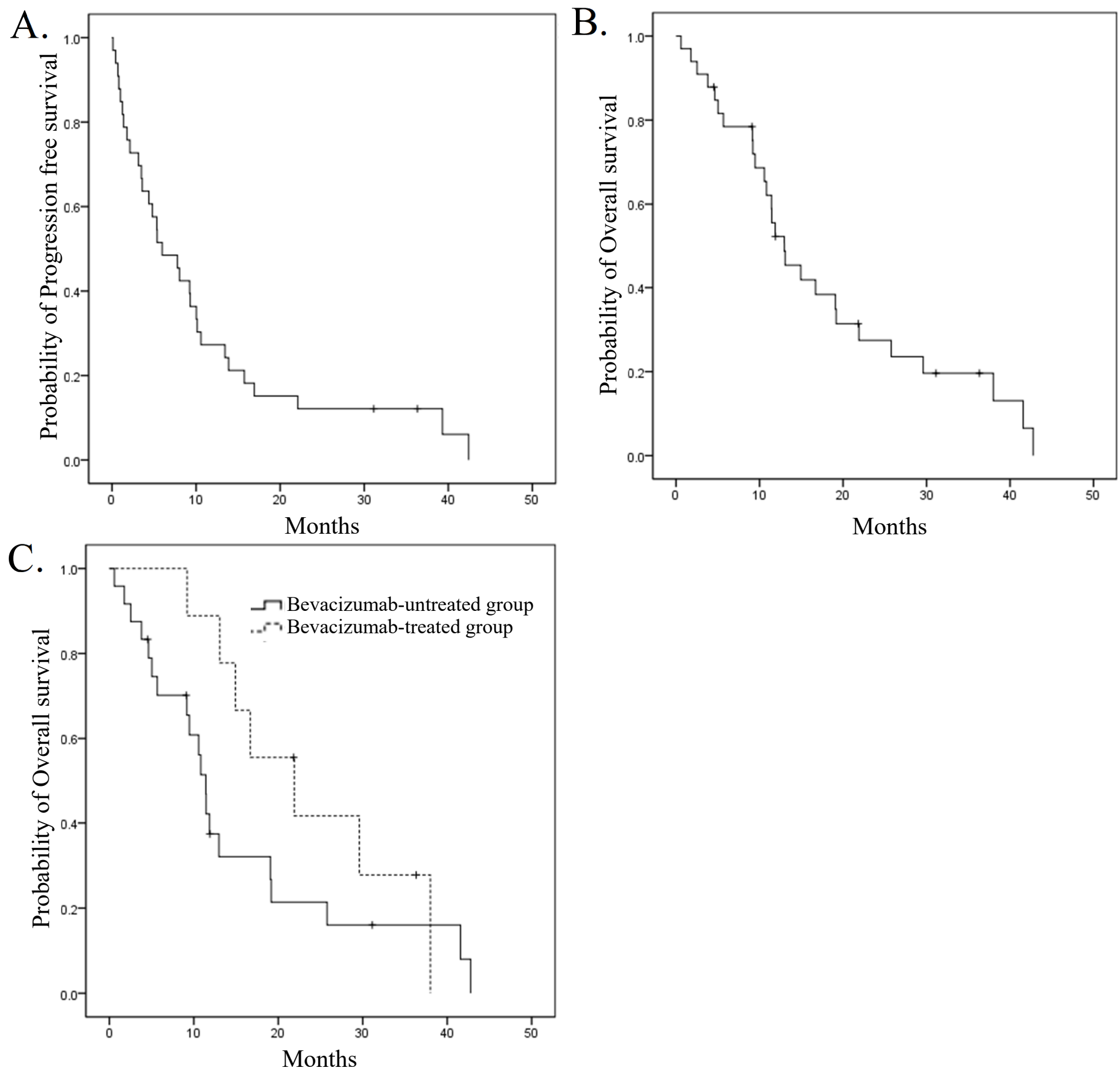

\section{Figure legends}

Figure 1. Kaplan-Meier plots for progression-free (PFS) and overall (OS) patient survival. (A) PFS curves for patients treated with first-line CT; (B) OS curves for patients treated with firstline CT; (C) OS curves for patients treated with or without the inclusion of bevacizumab. 